\title{
The role of Src homology 2 containing protein tyrosine phosphatase 2 in vascular smooth muscle cell migration and proliferation
}

\author{
Machender R KANDADI ${ }^{1,2}$, Matthew S STRATTON ${ }^{1}$, Jun REN ${ }^{1, *}$ \\ ${ }^{1}$ Center for Cardiovascular Research and Alternative Medicine \& Division of Pharmaceutical Sciences, University of Wyoming College \\ of Health Sciences, Laramie, WY 82071, USA; ${ }^{2}$ Department of Pharmacology, Manipal College of Pharmaceutical Sciences, Manipal \\ University, Manipal 576104, India
}

\begin{abstract}
Vascular smooth muscle cells (VSMCs) perform essential smooth muscle contractile and synthetic functions including migration, differentiation and proliferation under physiological and pathological conditions. In response to pathological stimuli, VMSCs undergo phenotypic change resulting in abnormal migration and proliferation, which may contribute to a "pathogenesis-like" atherosclerosis. Intracellular signaling mechanisms governing this phenotypic switch are of great significance not only for better understanding of atherosclerotic plaque formation but also for strategy for pertinent therapeutic remedies. Src Homology 2 Containing Protein Tyrosine Phosphatase 2 (SHP2) is a ubiquitous tyrosine phosphatase containing Src Homology 2 domains which plays major biological functions in response to various growth factors, hormones or cytokines. In particular, SHP2 is implicated in cell signaling pathways controlling cell cycle progression, growth and migration. In this review we will mainly discuss the recent literature demonstrating the role of SHP2 in VSMC migration and proliferation.
\end{abstract}

Keywords: Src homology 2 containing protein tyrosine phosphatase 2; vascular smooth muscle cells; migration; proliferation

Acta Pharmacologica Sinica (2010) 31: 1277-1283; doi: 10.1038/aps.2010.168; published online 27 Sep 2010

\section{Introduction}

Under physiological condition, vascular smooth muscle cells (VSMCs) exist in an organized, differentiated and contractile phenotype to perform contractile and synthetic functions characterized by changes in morphology, proliferation and migration rates. However, in response to pathological stimuli these contractile cells may be switched to a proliferative and migratory phenotype. The ability to shuttle between phenotypes is necessary for wound healing and growth of new vessels in developing tissues. On the other hand, VSMCs may contribute to pathological changes in the vasculature such as atherosclerosis when they are inappropriately stimulated to proliferate and migrate ${ }^{[1]}$. A plethora of efforts have been made attempting to elucidate the governing of this phenotypic switching in VSMCs. In particular, a wide variety of growth factors have been identified to participate in the regulation of VSMC proliferation and migration. For instance, VSMCs may migrate and proliferate with a fair degree of predictability in

\footnotetext{
* To whom correspondence should be addressed.

E-mail jren@uwyo.edu

Received 2010-05-26 Accepted 2010-08-27
}

response to platelet-derived growth factor $(\mathrm{PDGF})^{[2,3]}$. However, proliferation and migration can be bolstered or mitigated in VSMCs in the presence of other growth factors or stimuli. Thus the net outcome in proliferation and migration can be drastically different depending on the involvement of distinct signaling pathways ${ }^{[4]}$. Therefore a closer scrutiny of the cell signaling mechanisms seems essential in understanding the switch in phenotype for VSMC proliferation and migration. Among the many proteins and cell signaling molecules surfaced over the last decades, Src homology 2 (SH2) containing protein tyrosine phosphatase (PTP) 2 (SH-PTP2) has drawn some heavy attention recently in the regulation of multiple signaling pathways associated with growth and proliferation in a variety of cell types. SH-PTP2 is a member of the non receptor protein tyrosine phosphatase family and will be referred to as SHP2 in this review. SHP2, a 597 amino acid (AA) monomer first identified in human cells in 1992 as a homolog to the Drosophila Corkscrew protein ${ }^{[5]}$, acts by targeted dephosphorylation of substrate phosphotyrosine. The gene encoding this protein is found on chromosome 12 band q24.13. Mutations in this gene have been associated with several human genetic diseases such as Noonan syndrome, Leopard syndrome and 
Juvenile Myelo monocytic Leukemia ${ }^{[6]}$. Missense point mutations may result in increased phosphatase activity leading to short stature, facial dysmorphia, skeletal malformation and congenital heart disease in Noonan syndrome ${ }^{[7]}$. SHP2 also plays a significant role in intracellular signaling associated with growth factors, receptor tyrosine kinases, integrins, and immunoglobulin receptors in cell cycle, migration, proliferation and apoptosis regulation ${ }^{[5,8]}$.

\section{Structure and function}

Structurally, SHP2 is characterized by two tandem N terminal SH2 domains, a catalytic PTP domain and a C terminal tail. The two $\mathrm{SH} 2$ domains are named $\mathrm{N}$ and $\mathrm{C}$, with $\mathrm{N}$ being the $\mathrm{SH} 2$ domain closer to $\mathrm{N}$ terminal. These domains are about $100 \mathrm{AAs}$ in sequence and are found between AAs 6-101 (N-SH2) and 112-211 (C-SH2). The business end of the SHP2 stick, the PTP domain lies between AAs 276-523 and is followed by the C-terminal tail which extends up to AA $597^{[9]}$. To-date, several different clones are available from NCBI ranging from $460 \mathrm{AA}$ in length to the most prevalent $597 \mathrm{AA}$ clone.

SH2 domains are thought to localize SHP2 to phosphorylated tyrosine residues on substrate proteins. Based on chromatography affinity and peptide sequencing findings, Sweeney and colleagues predicted that there are 22 sequences the N-SH2 domain and 11 sequences that $\mathrm{C}-\mathrm{SH} 2$ domain bind with high affinity ${ }^{[10]}$. Lesser binding was observed in 150 and 77 sequences for $\mathrm{N}$ and C-SH2s, respectively. While C-SH2 exhibits affinity for $(\mathrm{T} / \mathrm{V} / \mathrm{I}) \mathrm{XpY}(\mathrm{A} / \mathrm{s} / \mathrm{t} / \mathrm{v}) \mathrm{X}(\mathrm{I} / \mathrm{v} / \mathrm{l})$ sequences, the N-SH2 domain shows affinity for 4 consensus sequences as the following: (i): (I/L/V/m) $\mathrm{XpY}(\mathrm{T} / \mathrm{V} / \mathrm{A}) \mathrm{X}(\mathrm{I} / \mathrm{V} / \mathrm{L} / \mathrm{f})$; (ii): $\mathrm{W}(\mathrm{M} / \mathrm{T} / \mathrm{v}) \mathrm{pY}(\mathrm{y} / \mathrm{r})(\mathrm{I} / \mathrm{L}) \mathrm{X}$; (iii): (I/V)XpY(L/M/T)Y(A/ $\mathrm{P} / \mathrm{T} / \mathrm{S} / \mathrm{g}$ ) and (iv): (I/V/L)XpY(F/M)XP. Note that items in parentheses indicate multiple AAs occurring with similar frequency at that location with lowercase letters representing less frequent AAs. In addition, $X$ represents no specificity at that location and $\mathrm{pY}$ is phosphorylated tyrosine ${ }^{[10]}$. Sequences iii and iv are conserved sequences (commonly observed N-SH2 domain substrates). Actually, binding of sequences $\mathrm{i}$ and ii has not been shown to occur naturally.

The PTP domain of SHP2 acts to dephosphorylate the tyrosine residues. PTP activity in SHP2 is negatively regulated primarily by a close association with the N-SH2 domain in the inactive form. Reversible oxidation may also serve as an additional negative regulator of the PTP domain at active serines ${ }^{[11]}$. The family of classical PTPs is characterized by the presence of 10 highly conserved motifs in their catalytic domains for structural conformation, substrate interaction and phosphates action. Differences in other AA sequences add another layer of variation thus allowing for more targeted PTP activity. It is believed that SHP2 dephosphorylates substrates in a manner distinctly from other PTPs due to its SH2 localization/targeting domains, and variation from other PTPs in the catalytic domain ${ }^{[6]}$. Several studies have examined the SHP2 catalytic domain substrate specificity using capture techniques although little information is available on the precise target sequences of the PTP domain of SHP2.
The C-terminal tail extending from AA 524 through the end is by far the most interesting and functionally elusive aspect of SHP-2. SHP2 possesses a generally positive effect on its substrate as opposed to its sister phosphatase SHP-1 which generally displays a negative effect on its substrate. While this statement may be somewhat too general, it does highlight the power of the C-tail as this is where most of the variations occur between SHP-1 and SHP2 to allow alternate function. For more detailed information regarding possible function of this domain, please see the review by Poole and Jones ${ }^{[12]}$. Within the SHP2 C-terminal, there are tyrosine and serine phosphorylation sites, membrane lipid interaction and lipid raft localization sites and proline rich domains (possible $\mathrm{SH} 3$ interaction sites $)^{[12]}$. When SHP2 is inactive the N-SH2 domain folds back to binds with the PTP sequence sequestering both domains although leaving the C-SH2 domain available for substrate interaction (Figure 1). This is thought to be the primary model of phosphatase regulation. When a phosphorylated substrate contacts the C-SH2 domain, the PTP/N-SH2 interaction is lost and $\mathrm{N}-\mathrm{SH} 2$ also binds the phosphorylated substrate or another in close proximity ${ }^{[13,14]}$. This allows the PTP domain to execute its phosphatase activity. Another more recent proposed method of activation is that the SHP2 tyrosine phosphorylation sites on the $\mathrm{C}$ terminal tail become phosphorylated and bind one or both $\mathrm{SH}-2$ domains thus freeing the PTP domain for activity ${ }^{[0]}$. Interestingly, the $\mathrm{C}$ terminal 543 tyrosine phosphorylation site is flanked by AAs that match an $\mathrm{SH} 2$ conserved affinity sequence. There has been much speculation with regards to how SHP2 activity is regulated and how $\mathrm{C}$ terminal phosphorylation affects localization and phosphatase activity of SHP2. SHP2 phosphorylation of tyrosine 542 and 580 has been shown as required for Grb 2 recruitment via its $\mathrm{SH} 2$ domain ${ }^{[12]}$.
A

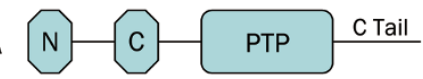

B

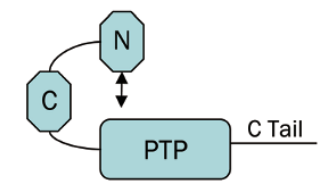

D
C
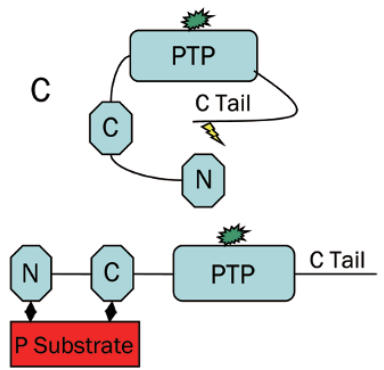

Figure 1. Possible structural conformations of SHP2. (A) Basic structure; (B) inactive form with N-SH2 and PTP domain interaction; (C) active form after $\mathrm{C}$ tail phosphorylation and $\mathrm{N}-\mathrm{SH} 2$ domain interaction; (D) active form after phosphorylated substrate binding of $\mathrm{N}$ and $\mathrm{C}-\mathrm{SH} 2$ domains.

Dephosphorylation of target molecules have potentially stimulatory or inhibitory affects on the overall signal transduction. For instance, phosphorylation at tyrosine 416 on Src kinase increases the kinase activity while phosphorylation at tyrosine 527 decreases the kinase activity. Dephosphorylation at these sites is expected to result in alternate outcomes ${ }^{[7]}$. 
Hence increased SHP2 phosphorylation may either increase or decrease the target signal molecules depending on microenvironment. Furthermore, if the role of SHP2 is to dephosphorylate a protein that inhibits the pathway then the net effect should be stimulatory. On the other hand, dephosphorylation might directly stimulate a substrate by creating a conformational change that opens a hidden binding site upon phosphorylation. The same relationship holds for possible negative regulation. Also, dephosphorylation of ubiquitination sites can positively reinforce expression of proteins that would otherwise be destroyed ${ }^{[15]}$. Receptor tyrosine kinases (the class of receptor that insulin growth factor receptor (IGFR), fibroblast growth factor receptor (FGFR), epidermal growth factor receptor (EGFR), and PDGF receptor belong to) have been shown to conduct signal transduction through activation of RAS/ MAPK, PI3K/AKT, and PLCY (Figure 2).

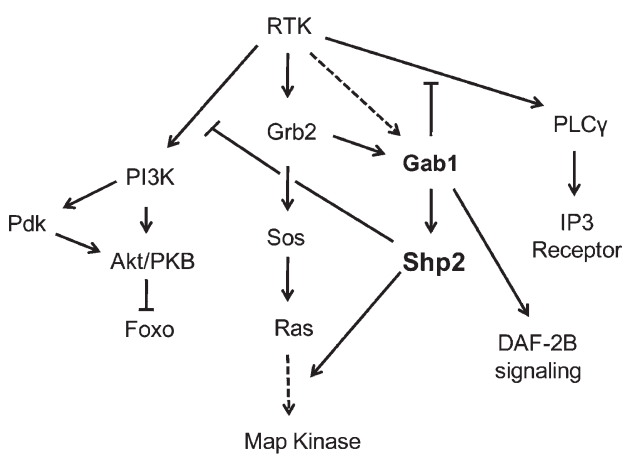

Figure 2. SHP2 in overall RTK receptor signaling. Adapted from Hopper $2006^{[16]}$.

\section{SHP2 and VSMCs}

Up-to-date, very few reports can be found on PubMed focusing on SHP2 in VSMCs. The majority of efforts have been engaged towards activation of immune system, control of migration and proliferation associated with cancers and insulin signaling. While all worthwhile endeavors, SHP2 is highly expressed in vasculature that the volume of research in other tissue types seems disproportionate. Here we will highlight signaling pathways where SHP2 has been implicated in VSMCs. In cases when little data are available in VSMCs, the analysis will be augmented with information of SHP2 in that specific pathway observed in other tissues or species.

\section{General expression profiles of SHP2 in VSMCs}

SHP2 is expressed widely throughout mammalian tissues although expression levels vary significantly among different tissues, with livers and kidneys displaying the least expression. Using immunohistochemical staining and in situ hybridization techniques, Adachi and coworkers have shown ubiquitous expression of SHP2 in VSMCs ${ }^{[17]}$. Seki and colleagues also reported elevated SHP2 expression in thickened intima in balloon-induced tissue remodeling ${ }^{[18]}$. Chang and coworkers observed a transient increase in SHP2 levels in medial
VSMCs after vascular injury. In addition they also reported that PDGF, basic fibroblast growth factor (bFGF) and nitric oxide (NO) but not Ang II, EGF and IGF-1 increase the SHP2 expression in cultured primary rat VSMC ${ }^{[18-20]}$. Recently, SHP2 expression and phosphorylation were found elevated in older VSMCs compared to the young ones ${ }^{[21]}$, indicating a possible role of SHP2 in vascular aging.

\section{SHP2 activity and signal transduction Growth factors and SHP2}

Growth factors such as platelet-derived growth factor (PDGF), epidermal growth factor (EGF), and insulin like growth factor-1 (IGF-1), were involved in smooth muscle cell migration and proliferation by stimulating various signaling mechanisms under physiological and pathological conditions.

PDGF induces SHP2 tyrosine phosphorylation. Tyrosine phosphorylation of SHP2 stimulates catalytic activity. The activated SHP2 binds directly to the activated PDGF receptor at phoshpotyrosine-1009 pY1009 via its N terminal domain and thereby dephosphorylates the receptor or its associated substrates $^{[22,23]}$. On the other hand, SHP2 is also implicated in positive regulation, since tyrosine phosphorylation of SHP2 presents a binding site for the adaptor protein Grb2. Grb2 then mediates Ras activation through complex formation with the nucleotide exchange factor Sos ${ }^{[23]}$. In addition SHP2 activity is also involved in phosphatidylinositol 3-kinase independent regulation of cell migration ${ }^{[24]}$.

IGF-1 induced smooth muscle cell migration or proliferation is mediated through $\alpha \mathrm{V} \beta 3$ integrin. IGF-1 stimulates $a V \beta 3$ integrin ligand occupancy and phosphorylation, which results in SHP2 recruitment to the plasma membrane. This membrane associated SHP2 is subsequently recruited to Src homology 2 domain-containing protein tyrosine phosphatase substrate-1 resulting in PTP-1 tyrosine phosphorylation ${ }^{[25,26]}$.

SHP-2 is a positive mediator of the mitogenic signal transduction induced by EGF. Tyrosine phosphorylation of the EGFR is followed by the recruitment of various $\mathrm{SH} 2$ domaincontaining intracellular proteins to the cytoplasmic domain of EGFR. Formation of a Shc-Grb2-sos sevenless complex and the activation of Ras are key events in the initiation of signals through activated EGFR ${ }^{[27]}$.

\section{SHP2 and focal adhesion kinase}

Focal adhesion kinase (FAK) is prototype for a family of non-receptor protein tyrosine kinase highly expressed in VSMCs $^{[28]}$. FAK is strongly activated by integrin, growth factor receptors and G-protein receptor engagement and serves to integrate downstream signals in response to a variety of ligands and agonists ${ }^{[29]}$. In particular, FAK is an intermediate signal in VSMC migration and proliferation ${ }^{[30]}$. Fibroblast SHP2 is required for cell spreading, migration and focal adhesion. FAK and SHP2-deficient cells exhibited similar phenotypes, suggesting that the turnover of focal adhesion sites required in migration is dependent on SHP2 action through cyclic activation and deactivation of FAK. In human VSMCs, 
SHP2 directly interacts with FAK by dephosphorylating FAK at Y397 and thereby regulating VSMC's focal adhesion and migration $^{[31,32]}$.

\section{SHP2 and Rho}

Rho functions as a molecular switch under the tight control of regulatory proteins that cycle between the active GTP-bound and inactive GDP-bound forms ${ }^{[33,34]}$. In middle passage rat VSMCs, peroxisome proliferator-activated receptors (PPAR) ligands suppresses Rho activity by up-regulating cytoplasmic SHP2 where it binds and inactivates VAV, a GTP/GDP exchange factor for Rho kinase ${ }^{[21,35]}$. In primary VSMCs, NO upregulates SHP2 expression (albeit not necessarily phosphorylation) and cell migration ${ }^{[36,37]}$. When SHP2 was attenuated via an antisense oligonucleotide, the NO-induced motility was decreased, possibly due to Rho A-dependent change in cell motility ${ }^{[37]}$. It was also shown that the NO-induced cell motility is largely due to the Gab1/SHP2 interaction. This raises the question regarding the precise nature of the Gab1/VAV interaction in proliferation and migration. When VSMCs are dedifferentiated, $\mathrm{NO}$ is capable of reducing motility ${ }^{[38]}$ and blocking the increased motility in response to PDGF treatment. However, after an extended period of culture in dedifferentiated cells with insulin, a Gab1/SHP2 interaction may lead to a decrease in Rho A abundance en route to the increased motility ${ }^{[39]}$. Rho is thought to be responsible for SHPS-1 phosphorylation which leads to SHP2 recruitment ${ }^{[40]}$. Depending on environment, Rho can be either up- or down-stream of SHP2.

\section{SHP2 and JAK/STAT}

The JAK/STAT pathway plays a rather significant role in vascular smooth muscle cell proliferation and migration induced by angiotensin II. In response to angiotensin II the JAK/STAT signaling in VSMCs involves the tyrosine phosphorylation and subsequent activation of JAK tyrosine kinases, which in turn phosphorylates STATs. The phosphorylated STAT translocates to nucleus as dimers and binds to specific response elements in gene promoters and direct transcription ${ }^{[41]}$. In middle passage rat VSMCs, SHP2 phosphorylation and localization on AT1 receptor are necessary for the JAK2/AT1 interaction and modulation of JAK2 downstream signaling ${ }^{[42]}$. On contrary Doan and colleagues have reported SHP2 as a negative regulator of angiotensin II signaling, where phosphorylated SHP2 was found to inhibit c-Src catalytic activity by dephosphorylating a positive regulatory tyrosine 418 within the Src kinase domain ${ }^{[43]}$. High glucose conditions augment the Ang II-induced ROS production, VSMC proliferation and tyrosine phosphorylation of JAK2 via the polyol pathway. The polyol pathway may in turn activate PKC $\beta 2$ and NADPH oxidase to generate more ROS. ROS is known to regulate the activity of SHP2. The altered state of SHP2 hinders its ability to interact with JAK2 $2^{[4]}$. In hyperglycemic states, SHP2 levels and activity display a two-fold increase in VSMCs compared with the normal glucose environment ${ }^{[45]}$.

\section{SHP2 and PI3K/AKT}

PI3-K is composed of a catalytic p110 subunit and a regulatory p85 subunit. PI3-K phosphorylates membrane glycerophospholipid phosphatidylinositol 4,5 biphosphate resulting in production of phosphatidylinositol 3,4,5 trisphosphate $\left(\mathrm{PIP}_{3}\right)$ and phosphatidylinositol 3,4 trisphosphate $\left(\mathrm{PIP}_{2}\right)$. PI3-K is an upstream signaling molecule of Akt which is also important in the maintenance of VSMC differentiation. SHP2 interacts with the p85 subunit of PI3-K and is a required step for IGF1 -induced PI3-K activation en route to cell migration and phosphorylation of Akt in middle passage VSMCs ${ }^{[25]}$. In EGFand Ang II-treated VSMCs, GAB 1 is rapidly phosphorylated and associates with the p85 subunit of PI3K and leads to Akt activation. When SHP2 is activated by resveratrol (compound from grape skin), it mitigates the GAB1/PI3K interaction and attenuates Akt activation ${ }^{[46]}$.

\section{SHP2 and integrins}

SHP2 recruitment to the plasma membrane is dependent on aV 33 integrin occupancy and phosphorylation. This SHP2 recruitment is also dependent on an insulin receptor substrate (IRS) family protein DAK1 which serves as a docking protein between SHP2 and the $\beta 3$ subunit of the integrin heterodimer $^{[47]}$. Once at the plasma membrane, it can be recruited to the SHPS-1, IRS-1, or other substrates. Transforming growth factor (TGF) $\beta$ stimulates the upregulation of aV $\beta 3$ integrins in 2-6 passage VSMCs. TGF $\beta$ is a positive proliferation effector of VSMC present following vascular injury ${ }^{[48]}$. aV $\beta 3$ integrins bind a non collagen substrate and are upregulated after oxidative damage in high passage proliferative cells ${ }^{[20]}$. Taken together, SHP2 recruitment to the plasma membrane by this specific integrin could be a primary mechanism priming VSMC migration and proliferation.

\section{SHP2 and MAPK}

SHP2 is tied to phenotype dependent response to IGF-1 in VSMCs. Upon IGF-1 stimulation, SHP2 dephosphorylated IRS-1 at Tyr-895 resulting in interrupted IRS-1/Grb 2/Sos signaling to Ras and subsequently ERK/p38 MAPK. Conversely after multiple passages in culture this negative regulation was silent and IGF-1 treatment led to IRS-1/Grb 2/Sos activation of Ras and subsequently of ERK/p38 MAPKs ${ }^{[4]}$. In middle passage VSMCs the GAB1 phosphorylation in response to PDGF was at least somewhat dependent on PKC and PLC $\gamma^{[49]}$. IGF-1-dependent Shc phosphorylation is required for MAPK activation and cell proliferation. Recruitment of SHP2 and Shc to SHPS-1 in response to IGF-1 are both required for Shc phosphorylation and therefore necessary for mediating IGF-1 dependent mitogenic signaling in $\mathrm{SMCs}^{[50]}$. On a separate note, unsaturated lysophosphatidic acid (LPA) is capable of transforming the primary differentiated VSMCs into dedifferentiated VSMCs via ERK and p38 MAPKs ${ }^{[51]}$. How does this unsaturated LPA affect SHP2? In Rat-1 cells, LPA was shown to increase SHP2 association with SHPS-1, representing a possible mechanism for VSMC dedifferentiation. The SHP2/ 
SHPS-1 interaction then appears to bring on MAPK activation at least in response to LPA and IGF-1. SHPS-1 is phosphorylated in the LPA and IGF-1 signaling ${ }^{[40]}$.

\section{Challenge in shp2 signaling in VSMCs}

SHP2 exerts negative effects on Akt in the EGF and Ang II signaling pathways whereas it elicits positive effects on MAPK in the IGF-1 and LPA signaling cascades. GAB is capable of recruiting SHP2 in both instances. In response to EGF and Ang II, GAB works in concert with the p85 subunit of PI3K. On the other side of the coin, GAB also associates with Grb 2 and the RTK signaling complex upon IGF-1 stimulation. In addition, factors favoring VSMC proliferation and migration seem to contribute to SHP-2 localization at the plasma membrane and its association with SHPS- 1 or other substrates, leading to subsequent MAPK activation. In increased migration due to SHP2 negative regulation of Rho, it is unclear where this takes place but again it looks as if there is SHP2-GAB interaction involved. Given that protein tyrosine phosphatase activity is often measured using dephosphorylation of $p$-nitrophenyl phosphate to offer an overall cellular phosphatase activity, it is often difficult to attribute the activity to a single phosphatase. Even with attempts being made to inhibit other phosphatases, the control is never adequate for all known dephosphorylation enzymes. Furthermore, it is not entirely clear if phosphorylation of C-terminal residues is a necessary step for phosphatase activity (possibly activated through SH2 domain interactions with substrate proteins). Further study with phospho residues in the C-tail of SHP2 as the marker for an active enzyme is warranted. It is difficult to sift through the seemingly contradictory evidence with regards to SHP2 activity and expression in VSMC action. Variables like cell type, cell age, growth factor employed, degree of confluence and time between treatment and assay must be considered in order to get a better picture of the vascular effect of SHP2. The complexity of SHP2 regulation and action further add to such technical difficulties.

With many possible catalytic, $\mathrm{SH} 2$ domain, and $\mathrm{C}$ tail substrates and regulators, SHP2 is clearly an important regulator of intracellular signaling. Co-precipitation assays and immunohistochemistry for cellular localization of molecules that alter phosphorylation states may also be used to determine possible interaction and site of action. Many further questions regarding the action of SHP2 remain. As a target for drug development, SHP2 is attractive because of its multiple sites and pathways for regulation. If the utility of the C terminal and SH2 domains can be better defined, possible antagonists or agonists for those specific regions may allow normal SHP2 action in a given pathway but "loss or gain of function" in another pathway. For instance if the section of the $\mathrm{C}$ tail responsible for phosphorylation and interaction with the SH2 domain is inactivated, one could limit SHP2 activity to targeted $\mathrm{N}$ and C-SH2 domain substrate interaction activation. Similarly, $\mathrm{C}$ terminal lipid raft localization motifs or the C-SH2 domain could be selectively inhibited to limit unique SHP2 action while preserving others. We should emphasize that any application will require a better understanding of the $\mathrm{SH} 2 / \mathrm{c}$-tail relationships.

In cases where SHP2 binding to a certain substrate is required for signal transduction, it is presumed that this signal is dependent on SHP2 phosphatase action on that substrate or one in the same complex. While some studies do connect SHP2 association with substrate protein and dephosphorylation of that or another substrate, most studies stop short. This may create a scenario that in cases where SHP2 is localized to its substrate proteins, it may actually be sequestered from other possible substrates.

\section{Acknowledgements}

We sincerely express our apology to those authors whose important work cannot be cited here due to space limitation.

\section{References}

1 Lundberg MS, Crow MT. Age-related changes in the signaling and function of vascular smooth muscle cells. Exp Gerontol 1999; 34: 549-57.

2 Bornfeldt KE, Raines EW, Nakano T, Graves LM, Krebs EG, Ross R. Insulin-like growth factor-I and platelet-derived growth factor-BB induce directed migration of human arterial smooth muscle cells via signaling pathways that are distinct from those of proliferation. J Clin Invest 1994; 93: 1266-74.

3 Hughes AD, Clunn GF, Refson J, Demoliou-Mason C. Platelet-derived growth factor (PDGF): actions and mechanisms in vascular smooth muscle. Gen Pharmacol 1996; 27: 1079-89.

4 Hayashi K, Shibata K, Morita T, Iwasaki K, Watanabe M, Sobue K. Insulin receptor substrate-1/SHP-2 interaction, a phenotypedependent switching machinery of insulin-like growth factor-I signaling in vascular smooth muscle cells. J Biol Chem 2004; 279: 40807-18.

5 Freeman RM Jr, Plutzky J, Neel BG. Identification of a human src homology 2-containing protein-tyrosine-phosphatase: a putative homolog of Drosophila corkscrew. Proc Natl Acad Sci USA 1992; 89: 11239-43.

6 Andersen JN, Mortensen OH, Peters GH, Drake PG, Iversen LF, Olsen $\mathrm{OH}$, et al. Structural and evolutionary relationships among protein tyrosine phosphatase domains. Mol Cell Biol 2001; 21: 7117-36.

7 Roskoski R Jr. Src kinase regulation by phosphorylation and dephosphorylation. Biochem Biophys Res Commun 2005; 331: 1-14.

8 Bennett AM, Tang TL, Sugimoto S, Walsh CT, Neel BG. Proteintyrosine-phosphatase SHPTP2 couples platelet-derived growth factor receptor beta to Ras. Proc Natl Acad Sci USA 1994; 91: 7335-9.

9 Neel BG, Gu H, Pao L. The 'Shp'ing news: SH2 domain-containing tyrosine phosphatases in cell signaling. Trends Biochem Sci 2003; 28: 284-93.

10 Sweeney MC, Wavreille AS, Park J, Butchar JP, Tridandapani S, Pei D. Decoding protein-protein interactions through combinatorial chemistry: sequence specificity of SHP-1, SHP-2, and SHIP SH2 domains. Biochemistry 2005; 44: 14932-47.

11 Kwon J, Qu CK, Maeng JS, Falahati R, Lee C, Williams MS. Receptorstimulated oxidation of SHP-2 promotes T-cell adhesion through SLP76-ADAP. Embo J 2005; 24: 2331-41.

12 Poole AW, Jones ML. A SHPing tale: perspectives on the regulation of SHP-1 and SHP-2 tyrosine phosphatases by the C-terminal tail. Cell Signal 2005; 17: 1323-32.

13 Pluskey S, Wandless TJ, Walsh CT, Shoelson SE. Potent stimulation 
of SH-PTP2 phosphatase activity by simultaneous occupancy of both SH2 domains. J Biol Chem 1995; 270: 2897-900.

14 Maheshwari M, Belmont J, Fernbach S, Ho T, Molinari L, Yakub I, et al. PTPN11 mutations in Noonan syndrome type I: detection of recurrent mutations in exons 3 and 13. Hum Mutat 2002; 20: 298-304.

15 Ali S, Nouhi Z, Chughtai N, Ali S. SHP-2 regulates SOCS-1-mediated Janus kinase-2 ubiquitination/degradation downstream of the prolactin receptor. J Biol Chem 2003; 278: 52021-31.

16 Hopper NA. The adaptor protein soc-1/Gab1 modifies growth factor receptor output in Caenorhabditis elegans. Genetics 2006; 173: 163-75.

17 Adachi M, Iwaki H, Shindoh M, Akao Y, Hachiya T, Ikeda M, et al. Predominant expression of the src homology 2-containing tyrosine phosphatase protein SHP2 in vascular smooth muscle cells. Virchows Arch 1997; 430: 321-5.

18 Seki N, Hashimoto N, Suzuki Y, Mori S, Amano K, Saito Y. Role of SRC homology 2-containing tyrosine phosphatase 2 on proliferation of rat smooth muscle cells. Arterioscler Thromb Vasc Biol 2002; 22: 1081-5.

19 Chang Y, Zhuang D, Zhang C, Hassid A. Increase of PTP levels in vascular injury and in cultured aortic smooth muscle cells treated with specific growth factors. Am J Physiol Heart Circ Physiol 2004; 287 : H2201-8.

20 Wilson E, Parrish AR, Bral CM, Williams ES, Ramos KS. Collagen suppresses the proliferative phenotype of allylamine-injured vascular smooth muscle cells. Atherosclerosis 2002; 162: 289-97.

21 Rice KM, Kinnard RS, Wright GL, Blough ER. Aging alters vascular mechanotransduction: pressure-induced regulation of p70S6 $\mathrm{k}$ in the rat aorta. Mech Ageing Dev 2005; 126: 1213-22.

22 Klinghoffer RA, Kazlauskas A. Identification of a putative Syp substrate, the PDGF beta receptor. J Biol Chem 1995; 270: 22208-17.

23 Li W, Nishimura R, Kashishian A, Batzer AG, Kim WJ, Cooper JA, et al. A new function for a phosphotyrosine phosphatase: linking GRB2-Sos to a receptor tyrosine kinase. Mol Cell Biol 1994; 14: 509-17.

24 Qi JH, Ito N, Claesson-Welsh L. Tyrosine phosphatase SHP-2 is involved in regulation of platelet-derived growth factor-induced migration. J Biol Chem 1999; 274: 14455-63.

25 Kwon M, Ling Y, Maile LA, Badley-Clark J, Clemmons DR. Recruitment of the tyrosine phosphatase Src homology 2 domain tyrosine phosphatase- 2 to the p85 subunit of phosphatidylinositol-3 (PI-3) kinase is required for insulin-like growth factor-l-dependent PI-3 kinase activation in smooth muscle cells. Endocrinology 2006; 147: 1458-65.

26 Ling Y, Maile LA, Clemmons DR. Tyrosine phosphorylation of the beta3-subunit of the alphaVbeta3 integrin is required for embrane association of the tyrosine phosphatase SHP-2 and its further recruitment to the insulin-like growth factor I receptor. Mol Endocrinol 2003; 17: 1824-33.

27 Montagner A, Yart A, Dance M, Perret B, Salles JP, Raynal P. A novel role for Gab1 and SHP2 in epidermal growth factor-induced Ras activation. J Biol Chem 2005; 280: 5350-60.

28 Polte TR, Naftilan AJ, Hanks SK. Focal adhesion kinase is abundant in developing blood vessels and elevation of its phosphotyrosine content in vascular smooth muscle cells is a rapid response to angiotensin II. J Cell Biochem 1994; 55: 106-19.

29 Mitra SK, Hanson DA, Schlaepfer DD. Focal adhesion kinase: in command and control of cell motility. Nat Rev Mol Cell Biol 2005; 6: 56-68.

30 Keffel S, Alexandrov A, Goepel M, Michel MC. alpha(1)-adrenoceptor subtypes differentially couple to growth promotion and inhibition in Chinese hamster ovary cells. Biochem Biophys Res Commun 2000; 272: 906-11.
31 Yu DH, Qu CK, Henegariu O, Lu X, Feng GS. Protein-tyrosine phosphatase Shp-2 regulates cell spreading, migration, and focal adhesion. J Biol Chem 1998; 273: 21125-31.

32 von Wichert G, Haimovich B, Feng GS, Sheetz MP. Force-dependent integrin-cytoskeleton linkage formation requires downregulation of focal complex dynamics by Shp2. Embo J 2003; 22: 5023-35.

33 Burridge K, Wennerberg K. Rho and Rac take center stage. Cell 2004; 116: 167-79.

34 Etienne-Manneville S, Hall A. Rho GTPases in cell biology. Nature 2002; 420: 629-35.

35 Wakino S, Hayashi K, Kanda T, Tatematsu S, Homma K, Yoshioka K, et al. Peroxisome proliferator-activated receptor gamma ligands inhibit Rho/Rho kinase pathway by inducing protein tyrosine phosphatase SHP-2. Circ Res 2004; 95: e45-55.

36 Brown C, Pan X, Hassid A. Nitric oxide and C-type atrial natriuretic peptide stimulate primary aortic smooth muscle cell migration via a cGMP-dependent mechanism: relationship to microfilament dissociation and altered cell morphology. Circ Res 1999; 84: 655-67.

37 Chang Y, Ceacareanu B, Dixit M, Sreejayan N, Hassid A. Nitric oxideinduced motility in aortic smooth muscle cells: role of protein tyrosine phosphatase SHP-2 and GTP-binding protein Rho. Circ Res 2002; 91 : 390-7.

38 Sarkar R, Meinberg EG, Stanley JC, Gordon D, Webb RC. Nitric oxide reversibly inhibits the migration of cultured vascular smooth muscle cells. Circ Res 1996; 78: 225-30.

39 Dixit M, Zhuang D, Ceacareanu B, Hassid A. Treatment with insulin uncovers the motogenic capacity of nitric oxide in aortic smooth muscle cells: dependence on Gab1 and Gab1-SHP2 association. Circ Res 2003; 93: e113-23.

40 Takeda H, Matozaki T, Fujioka Y, Takada T, Noguchi T, Yamao T, et al. Lysophosphatidic acid-induced association of SHP-2 with SHPS1: roles of RHO, FAK, and a SRC family kinase. Oncogene 1998; 16 : 3019-27.

41 Schindler C, Darnell JE Jr. Transcriptional responses to polypeptide ligands: the JAK-STAT pathway. Annu Rev Biochem 1995; 64: 621-51.

42 Marrero MB, Venema VJ, Ju H, Eaton DC, Venema RC. Regulation of angiotensin II-induced JAK2 tyrosine phosphorylation: roles of SHP-1 and SHP-2. Am J Physiol 1998; 275: C1216-23.

43 Doan T, Farmer P, Cooney T, Ali MS. Selective down-regulation of angiotensin II receptor type $1 \mathrm{~A}$ signaling by protein tyrosine phosphatase SHP-2 in vascular smooth muscle cells. Cell Signal 2004; 16: 301-11.

44 Shaw S, Wang X, Redd H, Alexander GD, Isales CM, Marrero MB. High glucose augments the angiotensin II-induced activation of JAK2 in vascular smooth muscle cells via the polyol pathway. J Biol Chem 2003; 278: 30634-41.

45 Amiri F, Venema VJ, Wang X, Ju H, Venema RC, Marrero MB. Hyperglycemia enhances angiotensin II-induced janus-activated kinase/ STAT signaling in vascular smooth muscle cells. J Biol Chem 1999; 274: 32382-6.

46 Haider UG, Roos TU, Kontaridis MI, Neel BG, Sorescu D, Griendling KK, et al. Resveratrol inhibits angiotensin II- and epidermal growth factormediated Akt activation: role of Gab1 and Shp2. Mol Pharmacol 2005; 68: 41-8.

47 Ling Y, Maile LA, Badley-Clarke J, Clemmons DR. DOK1 mediates SHP2 binding to the alphaVbeta3 integrin and thereby regulates insulinlike growth factor I signaling in cultured vascular smooth muscle cells. J Biol Chem 2005; 280: 3151-8.

48 Li JM, Fan LM, Shah A, Brooks G. Targeting alphavbeta3 and alpha5beta1 for gene delivery to proliferating VSMCs: synergistic 
effect of TGF-beta1. Am J Physiol Heart Circ Physiol 2003; 285: H1123-31.

49 Saito Y, Hojo Y, Tanimoto T, Abe J, Berk BC. Protein kinase C-alpha and protein kinase $\mathrm{C}$-epsilon are required for Grb2-associated binder-1 tyrosine phosphorylation in response to platelet-derived growth factor. J Biol Chem 2002; 277: 23216-22.

50 Ling Y, Maile LA, Lieskovska J, Badley-Clarke J, Clemmons DR. Role of
SHPS-1 in the regulation of insulin-like growth factor l-stimulated Shc and mitogen-activated protein kinase activation in vascular smooth muscle cells. Mol Biol Cell 2005; 16: 3353-64.

51 Hayashi K, Takahashi M, Nishida W, Yoshida K, Ohkawa Y, Kitabatake A, et al. Phenotypic modulation of vascular smooth muscle cells induced by unsaturated lysophosphatidic acids. Circ Res 2001; 89: 251-8. 